Article

\title{
Pharmacokinetics of Tedizolid and Pseudoephedrine Administered Alone or in Combination in Healthy Volunteers
}

\author{
Shawn Flanagan ${ }^{1} *$, Sonia L. Minassian ${ }^{2}$ and Philippe Prokocimer ${ }^{1}$ \\ 1 Merck \& Co., Inc., Kenilworth, NJ 07033, USA; ppkprokocimer@gmail.com \\ 2 Minassian Biostatistics, Inc., San Diego, CA 92121, USA; sminassi@yahoo.com \\ * Correspondence: sf.in.sd@earthlink.net; Tel.: +1-858-249-7686
}

Received: 6 June 2018; Accepted: 13 June 2018; Published: 14 June 2018

\begin{abstract}
Therapeutic doses of tedizolid phosphate, an oxazolidinone antibiotic, lack monoamine oxidase inhibition invivo, potentially resulting in an improved safety profile versus other oxazolidinones. This randomized, double-blind, placebo-controlled, 2-period, 2-sequence, crossover, phase 1 study (NCT01577459) assessed the potential for pharmacokinetic (PK) interactions between tedizolid and pseudoephedrine. Eighteen healthy volunteers (age: 18-45 years) were block-randomized to 1 of 2 treatment sequences containing 2 treatment periods (tedizolid phosphate or placebo once daily for 4 days; single dose of pseudoephedrine $60 \mathrm{mg}$ on day 5) separated by a 2-day washout. Median time to maximum plasma concentration for tedizolid and pseudoephedrine ranged from 3 to $4 \mathrm{~h}$, regardless of treatment coadministration. Steady-state tedizolid had no effect on the PK of pseudoephedrine; geometric mean ratio and 90\% confidence interval remained within the no-effect 0.8 to 1.25 boundaries. The maximum observed concentration of tedizolid decreased by approximately $14 \%$ when pseudoephedrine was coadministered; no changes in the area under the plasma concentration-time curve or the minimum observed plasma concentration occurred. All adverse events (AEs) were mild, and there were no serious AEs or study drug discontinuations. No meaningful PK interactions occurred between tedizolid and pseudoephedrine, and tedizolid was well tolerated when administered in conjunction with pseudoephedrine.
\end{abstract}

Keywords: antibiotics; drug interactions; oxazolidinone; infectious disease; acute bacterial skin infections; pharmacokinetics

\section{Introduction}

Agents approved for the treatment of acute bacterial skin and skin structure infections (ABSSSI) are generally efficacious, but toxicity and microbial resistance may limit their use [1,2]. Given the increasing prevalence of multidrug-resistant, gram-positive pathogens, there is a need for new and effective antibiotics with proven antibacterial activity [2,3]. Oxazolidinones are one such class of antibacterial agents.

The oxazolidinone antibiotic linezolid, an inhibitor of monoamine oxidase (MAO), is approved for the treatment of uncomplicated and complicated skin and skin structure infections; however, significant safety concerns have emerged since its approval [4]. Coadministration of linezolid with other MAO inhibitors, adrenergic drugs, or serotonergic drugs is restricted because it may produce cardiovascular (e.g., hypertension) or neurologic (i.e., serotonin syndrome) treatment-emergent adverse events (AEs) that can be fatal in some patients [3,4]. The potential severity of these complications restricts investigation of the interaction in patients for ethical reasons; preclinical and healthy volunteer (phase 1) studies are used to better control the experimental parameters. Additionally, clinical studies have 
demonstrated that linezolid increases blood pressure in patients who have uncontrolled hypertension and in those who are taking sympathomimetic drugs such as pseudoephedrine [4,5], which are associated with increases in systolic blood pressure (SBP) and heart rate (HR) [6].

Tedizolid phosphate, a novel oxazolidinone antibiotic approved for the treatment of ABSSSI (200 mg once daily for 6 days) [7,8], demonstrated noninferiority compared with linezolid (600 mg twice daily for 10 days) in 2 randomized controlled phase 3 clinical trials (ESTABLISH-1 and ESTABLISH-2) $[9,10]$. Tedizolid, like linezolid, is a reversible inhibitor of MAO type A (MAO-A) and type B (MAO-B) in vitro [7,8]. Unlike linezolid, however, therapeutic doses of tedizolid phosphate lack MAO inhibition in vivo and subsequent hypertensive adrenergic or serotonergic adverse consequences, as reported in nonclinical and clinical studies in healthy volunteers [11].

This report presents the pharmacokinetic (PK) data that were generated during the phase 1 study (NCT01577459). The pharmacodynamic data (PD) from this same study were previously reported and showed a lack of PD interaction between tedizolid phosphate and pseudoephedrine, in that no significant difference was detected in the maximum change in SBP of healthy volunteers who either received pseudoephedrine with tedizolid phosphate compared with those who received pseudoephedrine with placebo $(11.6 \mathrm{mmHg}$ vs. $12.1 \mathrm{mmHg} ; p=0.73)$ [11]. Specifically, only $4(22 \%)$ of the participants receiving pseudoephedrine with tedizolid phosphate experienced an increase in SBP of $\geq 15 \mathrm{mmHg}$, while 5 (28\%) of the participants who received pseudoephedrine with placebo experienced the same SBP increase. Similarly, no significant difference was found between the maximum HR changes in the group receiving pseudoephedrine with tedizolid phosphate compared with those who received pseudoephedrine with placebo ( 13.6 beats/min vs. 15.2 beats $/ \mathrm{min}$, respectively; $p=0.17$ ).

To ensure that no underlying PK differences confounded the previously reported PD assessment, here we assess the potential for PK interactions between tedizolid phosphate and pseudoephedrine [11]. As a single agent, the PK of tedizolid phosphate has been characterized [7]. The prodrug, tedizolid phosphate, is rapidly cleaved into the active moiety, tedizolid following oral and intravenous administration, and has a half-life of approximately $12 \mathrm{~h}$. Steady-state concentrations of tedizolid are reached in approximately 3 days and tedizolid accumulation is approximately $30 \%$. In plasma, tedizolid accounts for $95 \%$ of the total radiocarbon AUC and there are no other circulating metabolites. The majority of the dose is metabolized to an inactive sulfate conjugate and is eliminated via the liver with approximately $82 \%$ appearing in the feces and $18 \%$ in urine [12]. The PK profile of pseudoephedrine as a single agent has also been documented. The half-life of pseudoephedrine

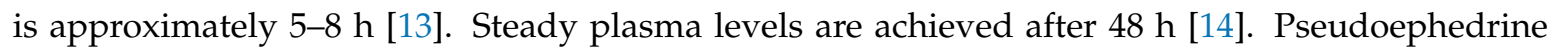
is primarily eliminated unchanged in the urine with small amounts of a hepatic metabolite. While minimal or no PK interaction between tedizolid and pseudoephedrine would be expected, we sought to confirm the absence of a yet unknown mechanism.

\section{Experimental Section}

\subsection{Ethics}

This study (ClinicalTrials.gov registration number NCT01577459) was conducted by Vince and Associates Clinical Research (Overland Park, KS, USA) in accordance with the ethical principles that have their origin in the Declaration of Helsinki and are consistent with Good Clinical Practice and with applicable national and institutional regulatory requirements. The study protocol (sponsor reference TR701-114) was approved by the MidLands Independent Review Board (Overland Park, KS, USA), and all participants provided written informed consent before any study procedures were performed.

\subsection{Study Design and Treatments}

This randomized, double-blind, placebo-controlled, 2-period, 2-sequence, crossover, phase 1 study was designed to evaluate the effects of steady state $200 \mathrm{mg}$ once-daily oral tedizolid phosphate or placebo on single-dose PK of pseudoephedrine in healthy participants. Steady-state PK of 
tedizolid, the biologically active moiety of tedizolid phosphate, with and without pseudoephedrine administration, was also determined.

Participants $(N=18)$ were block-randomized to 1 of 2 treatment sequences, with each sequence containing 2 treatment periods separated by a 2-day washout. During each treatment period, tedizolid phosphate or placebo was administered once daily for 4 days to reach steady state. On day 5 of each treatment period, a single dose of immediate-release pseudoephedrine $60 \mathrm{mg}$ was administered simultaneously with the study drug.

\subsection{Participants}

Participants included male and female healthy volunteers aged 18 to 45 years whose body mass index (BMI) ranged from $\geq 19.0 \mathrm{~kg} / \mathrm{m}^{2}$ to $\leq 31.0 \mathrm{~kg} / \mathrm{m}^{2}$, and met the following criteria: no clinically significant abnormalities identified by a detailed medical history, complete physical examination, 12-lead electrocardiograms and clinical laboratory tests, and negative pregnancy test results at screening and on study day -1 in females of childbearing potential.

Exclusion criteria were clinically significant history or evidence of cardiovascular, respiratory, hepatic, renal, gastrointestinal, endocrine, neurologic, immunologic, or psychiatric disorder(s); history of alcohol or substance abuse; SBP $>130 \mathrm{mmHg}$ or $<90 \mathrm{mmHg}$; diastolic blood pressure (DBP) $>90 \mathrm{mmHg}$ or $<60 \mathrm{mmHg}$; HR $>90 \mathrm{bpm}$ or $<50 \mathrm{bpm}$ after $10 \mathrm{~min}$ supine at screening and on study day -1 ; and QT interval corrected for HR using Fridericia's formula $>500 \mathrm{~ms}$. Previous or concomitant use of prescription or nonprescription drugs, herbal supplements, illicit drugs, or tobacco ( $\geq 10$ cigarettes/day) or nicotine-containing products was prohibited during the study. Diets high in tyramine or containing alcohol, grapefruit, caffeine, or xanthine were also prohibited. Previous participants in a tedizolid phosphate or tedizolid clinical study were excluded.

\subsection{Measurements}

Blood samples were collected at $0,1,2,3,4,6,8,12$, and $24 \mathrm{~h}$ after study drug administration on study days 4 and 5 . The PK of tedizolid, assessed on day 4 , was compared with the PK of tedizolid assessed on day 5 with the coadministration of pseudoephedrine. Pseudoephedrine PK on day 5 was compared between treatment periods (administration with tedizolid phosphate or placebo).

Throughout the study, safety assessments were collected, and these included vital signs, AEs, clinical laboratory evaluations, and physical examinations.

Participants were discharged from the clinic on study day 13, and follow-up assessments were conducted 7 days ( \pm 1 day) after discharge.

\subsection{Statistical Analyses}

The intent-to-treat (ITT) analysis set included all participants who gave informed consent and were randomly assigned to a treatment sequence. The PK analysis set included participants who received at least 1 administration of pseudoephedrine or tedizolid phosphate and had at least 1 postadministration collection of blood. The safety analysis set included participants who received any study drug.

Participant disposition, baseline demographics, and safety outcomes were summarized using descriptive characteristics. PK parameters evaluated included area under the plasma concentration-time curve from hour 0 to the last quantifiable time point $\left(\mathrm{AUC}_{0-\mathrm{t}}\right), \mathrm{AUC}_{0-24}, \mathrm{AUC}_{0-\infty}$, maximum observed concentration $\left(\mathrm{C}_{\max }\right)$, minimum observed concentration $\left(\mathrm{C}_{\min }\right)$ for tedizolid only, and time to maximum observed concentration $\left(\mathrm{T}_{\max }\right)$. Mean PK values were compared between the 2 treatments using analysis of variance (ANOVA) models with the log of the PK parameter as the dependent variable, treatment as a fixed factor, and participant as a random factor. Results from the ANOVA model were used to generate geometric mean (GM) values to calculate a GM ratio (GMR) for each parameter, and a $90 \%$ confidence interval (CI) for each GMR. 


\section{Results}

\subsection{Baseline Characteristics}

All participants met the criteria for all analysis sets, and thus, the ITT, PK, and safety analysis sets were identical and included data from all 18 randomly assigned participants (9 per treatment sequence). Most (83.3\%) participants were men, 50\% were white, and $44.4 \%$ were black; their mean ( \pm standard deviation) age was $34.4( \pm 7.5)$ years, and their mean BMI was $26.7( \pm 2.5) \mathrm{kg} / \mathrm{m}^{2}$.

\subsection{Pharmacokinetics}

Median $\mathrm{T}_{\max }$ values for tedizolid and pseudoephedrine were similar ( 3 to $4 \mathrm{~h}$ ) regardless of coadministration (data not shown). Steady state tedizolid had no effect on the PK of pseudoephedrine; the GMR and $90 \%$ CIs remained within the no-effect 0.8 to 1.25 boundaries (Table 1) [15]. $C_{\max }$ of tedizolid decreased by approximately $14 \%$ when pseudoephedrine was coadministered; no changes in AUC or $C_{\text {min }}$ were observed, as indicated by the GMR and 90\% CIs remaining between 0.8 and 1.25 (Table 1). Most participants had similar tedizolid PK exposure with and without pseudoephedrine (Figure 1A) and similar pseudoephedrine exposure with and without tedizolid phosphate (Figure 1B).

Table 1. Effects on plasma PK parameters (PK analysis set).

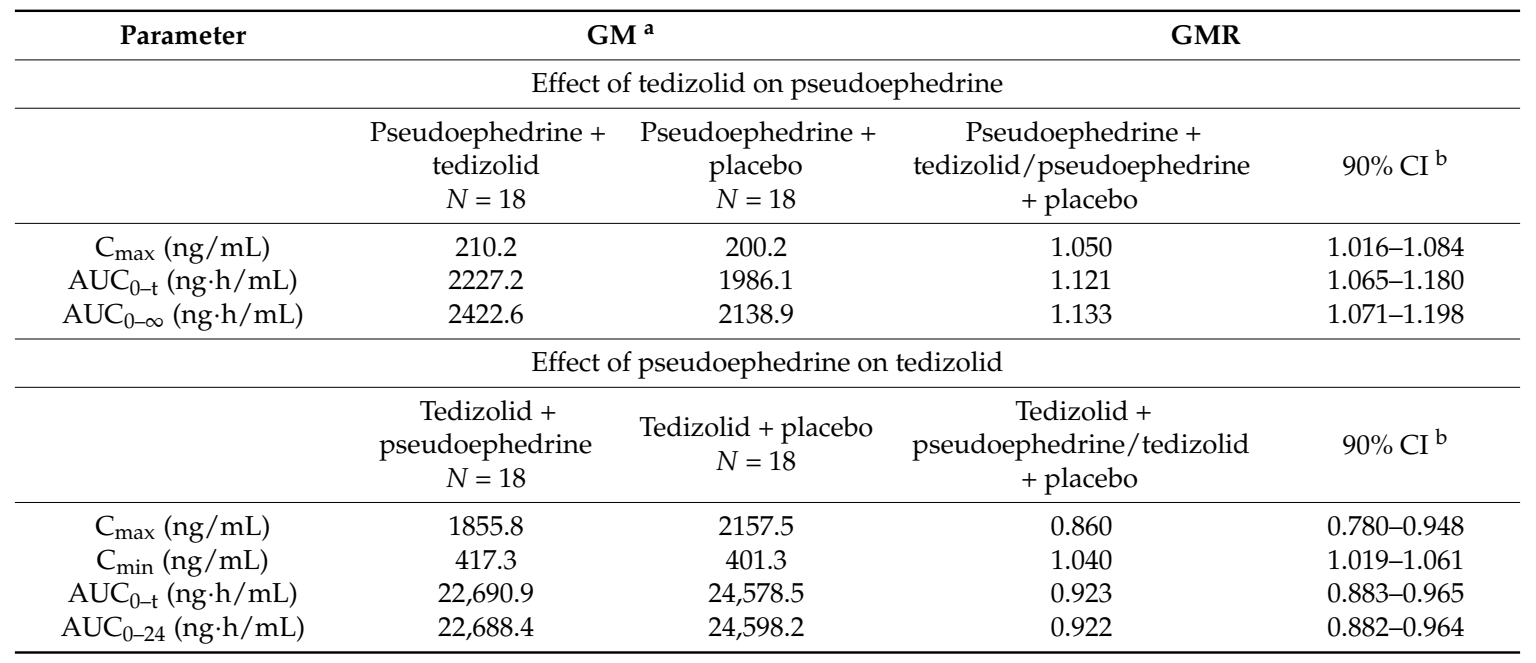

a Least squares mean from ANOVA model, calculated by transforming the natural log mean back to the linear scale (i.e., geometric mean). The ANOVA model included the log of the PK parameter as the response, treatment as a fixed factor, and participant as a random factor; ${ }^{b} 90 \%$ CI for the GMR. Abbreviations: ANOVA, analysis of variance; $\mathrm{AUC}_{0-\mathrm{t}}$, area under the plasma concentration-time curve from hour 0 to last quantifiable time point; $\mathrm{AUC}_{0-24}$, area under the plasma concentration-time curve from hour 0 to hour 24 ; $\mathrm{AUC} \mathrm{C}_{0-\infty}$, area under the plasma concentration-time curve from hour 0 extrapolated to infinity based on the apparent terminal rate constant; $\mathrm{CI}$, confidence interval; $\mathrm{C}_{\max }$, maximum observed concentration; $\mathrm{C}_{\min }$, minimum observed concentration; $\mathrm{GM}$, geometric mean; GMR, geometric mean ratio; PK, pharmacokinetic. 

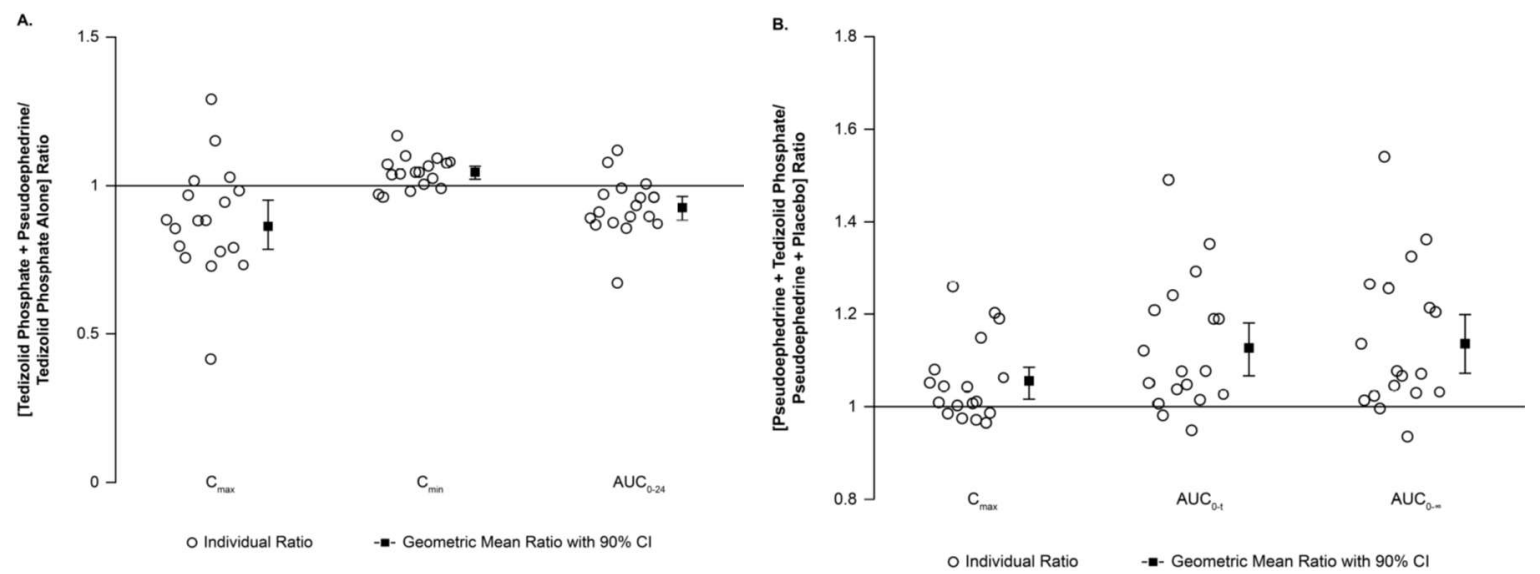

Figure 1. By-participant display of PK parameter ratios. (A) Individual tedizolid $\mathrm{C}_{\max }$, $\mathrm{C}_{\text {min }}$, and $\mathrm{AUC}_{0-24}$ treatment ratios (tedizolid + pseudoephedrine/tedizolid alone), GMRs, and corresponding 90\% CIs; (B) Individual pseudoephedrine $\mathrm{C}_{\max }, \mathrm{AUC}_{0-\mathrm{t}}$, and $\mathrm{AUC}_{0-\infty}$ treatment ratios (pseudoephedrine + tedizolid/pseudoephedrine + placebo), GMRs, and corresponding 90\% CIs. Abbreviations: $\mathrm{AUC}_{0-\mathrm{t}}$, area under the plasma concentration-time curve from hour 0 to last quantifiable time point; $\mathrm{AUC}_{0-24}$, area under the plasma concentration-time curve from hour 0 to hour 24; $\mathrm{AUC}_{0-\infty}$, area under the plasma concentration-time curve from hour 0 extrapolated to infinity based on the apparent terminal rate constant; $\mathrm{CI}$, confidence interval; $\mathrm{C}_{\max }$, maximum observed concentration; $\mathrm{C}_{\mathrm{min}}$, minimum observed concentration; $\mathrm{GM}$, geometric mean; $\mathrm{GMR}$, geometric mean ratio; PK, pharmacokinetic.

\subsection{Safety}

Overall, tedizolid phosphate $200 \mathrm{mg}$ for 5 days plus pseudoephedrine on study day 5 was well tolerated. Similar rates of AEs were reported during the placebo treatment period $(N=5)$ and the tedizolid phosphate treatment period $(N=4)$, and all AEs occurred after the first administration of pseudoephedrine (data not shown). All AEs were mild, none were serious, and no participant discontinued the study drug. Only headache and blood bilirubin increase were reported for more than 1 participant. Headaches occurred in 1 participant receiving placebo and 1 participant receiving tedizolid phosphate. Elevated bilirubin was found in 1 participant during both treatment periods and 1 participant only during treatment with tedizolid phosphate. Throughout the study, no notable laboratory or physical findings were observed.

Common AEs associated with pseudoephedrine include dry mouth, nausea, dizziness, insomnia, and nervousness [13]. Common AEs associated with tedizolid include nausea, headache, diarrhea, vomiting, and dizziness [7].

\section{Discussion}

These phase 1 study results demonstrated that there was no PK drug-drug interaction between tedizolid and pseudoephedrine. The PK of pseudoephedrine was unchanged when it was coadministered with tedizolid phosphate at steady state. No meaningful changes in tedizolid PK were noted with pseudoephedrine coadministration: $C_{\max }$ showed a slight decrease (14\%) when pseudoephedrine was coadministered, whereas AUC values (the key determinant for efficacy) and $\mathrm{C}_{\min }$ (related to safety) were unchanged. Plasma concentrations of tedizolid and pseudoephedrine each reached $C_{\max }$ at approximately the same time. Hence, the lack of pharmacologic interaction with coadministration cannot be explained by the drug administration scheduling.

These data confirm previously published results demonstrating that in various experimental settings, reflecting adrenergic and serotonergic systems, there were no MAO-mediated PD interactions between tedizolid and pseudoephedrine [11]. In contrast to the increase in SBP and DBP observed 
with linezolid [5], coadministration of tedizolid phosphate and pseudoephedrine did not significantly affect SBP, DBP, or HR [11]. The lack of MAO inhibition observed with tedizolid phosphate in vivo suggests an improved safety profile compared with linezolid, presenting a meaningful advantage in the many patients taking adrenergic or serotonergic agents concomitantly. A plausible explanation for the lack of in vivo drug-drug interaction is that free tedizolid $C_{\max }$ concentrations are consistently well below the tedizolid half-maximal inhibitory concentration $\left(\mathrm{IC}_{50}\right)$ for MAO (at $20 \%$ of MAO $\mathrm{IC}_{50}$ ) inhibition, a standard marker of pharmacologic activity.

Although generally well tolerated as a class, prolonged use of oxazolidinones, particularly linezolid, has been associated with reversible myelosuppression, specifically thrombocytopenia [4]. An assessment of 2 phase 3 clinical trials (ESTABLISH-1 and ESTABLISH-2) that compared tedizolid with linezolid in the treatment of patients with acute bacterial skin and skin structure infections (ABSSSIs) showed that 6 days of $200 \mathrm{mg}$ once daily tedizolid was associated with a lower incidence of thrombocytopenia versus 10 days of $600 \mathrm{mg}$ linezolid twice daily ( $3.2 \%$ and $5.6 \%$, respectively) [16]. The ESTABLISH-1 and ESTABLISH-2 trials confirmed this tedizolid dosing regimen as non-inferior to the linezolid regimen, which was both longer and uses a higher drug concentration $[9,10]$. In vitro susceptibility studies demonstrate that the minimum inhibitory concentration (MIC) of tedizolid is lower than that of linezolid for Staphylococcus aureus ( $\mathrm{MIC}_{90}$ of $0.25 \mu \mathrm{g} / \mathrm{mL}$ vs. $2 \mu \mathrm{g} / \mathrm{mL}$ ), coagulase-negative staphylococci (MIC 90 of $0.25 \mu \mathrm{g} / \mathrm{mL}$ vs. $2 \mu \mathrm{g} / \mathrm{mL}$ ), as well as several other isolates including viridans group streptococci and beta-hemolytic streptococci [17]. The difference in concentration and duration of therapeutic dose of tedizolid and linezolid may contribute to this and other findings that suggest tedizolid has a favorable safety profile while offering non-inferior efficacy compared with linezolid in patients with ABSSSIs [9-11,16,18].

\section{Conclusions}

In conclusion, these results demonstrate that no meaningful PK interactions occur between tedizolid and pseudoephedrine, supporting previously published results on lack of an MAO PD interaction. Tedizolid was well tolerated when administered with pseudoephedrine.

Author Contributions: S.F. contributed to the conception and design of the study, acquisition of data, analysis of data, and writing and critical review or revision of the manuscript. S.L.M. contributed to the analysis of the data and to the critical review or revision of the manuscript. P.P. contributed to the conception and design of the study, interpretation of results, and critical review or revision of the manuscript. In collaboration with the authors, employees of the study sponsor were involved in study design, data analysis, interpretation of results, and review of the manuscript.

Funding: This work was supported by Merck \& Co., Inc., Kenilworth, NJ, USA.

Acknowledgments: Medical writing and editorial assistance was provided by Sally Mitchell, and Sarah Utley, of ApotheCom, Yardley, PA. Editorial support was funded by Merck \& Co., Inc., Kenilworth, NJ, USA.

Conflicts of Interest: S.F. was an employee of Merck at the time of the study. P.P. was an employee and a stockholder of Merck at the time of the study. S.L.M. is a consultant to Merck and was compensated for supporting this research. The authors have no other funding or conflicts of interest to disclose.

\section{References and Notes}

1. Stevens, D.L.; Bisno, A.L.; Chambers, H.F.; Dellinger, E.P.; Goldstein, E.J.; Gorbach, S.L.; Hirschmann, J.V.; Kaplan, S.L.; Montoya, J.G.; Wade, J.C. Infectious Diseases Society of America. Practice guidelines for the diagnosis and management of skin and soft tissue infections: 2014 update by the Infectious Diseases Society of America. Clin. Infect. Dis. 2014, 59, e10-e52. [CrossRef] [PubMed]

2. Shaw, K.J.; Barbachyn, M.R. The oxazolidinones: Past, present, and future. Ann. N. Y. Acad. Sci. 2011, 1241, 48-70. [CrossRef] [PubMed]

3. Vinh, D.C.; Rubinstein, E. Linezolid: A review of safety and tolerability. J. Infect. 2009, 59 (Suppl. 1), S59-S74. [CrossRef]

4. Zyvox (linezolid); Package Insert; Pharmacia \& Upjohn Co., Division of Pfizer Inc.: New York, NY, USA, 2017. 
5. Hendershot, P.E.; Antal, E.J.; Welshman, I.R.; Batts, D.H.; Hopkins, N.K. Linezolid: Pharmacokinetic and pharmacodynamic evaluation of coadministration with pseudoephedrine $\mathrm{HCl}$, phenylpropanolamine $\mathrm{HCl}$, and dextromethorpan HBr. J. Clin. Pharmacol. 2001, 41, 563-572. [CrossRef] [PubMed]

6. Salerno, S.M.; Jackson, J.L.; Berbano, E.P. Effect of oral pseudoephedrine on blood pressure and heart rate: A meta-analysis. Arch. Intern. Med. 2005, 165, 1686-1694. [CrossRef] [PubMed]

7. Sivextro (tedizolid phosphate); Package Insert; Merck \& Co., Inc.: Whitehouse Station, NJ, USA, 2017.

8. Sivextro (tedizolid phosphate); Summary of Product Characteristics; Merck Sharp \& Dohme Limited: Hertfordshire, UK, 2015.

9. Moran, G.J.; Fang, E.; Corey, G.R.; Das, A.F.; De Anda, C.; Prokocimer, P. Tedizolid for 6 days versus linezolid for 10 days for acute bacterial skin and skin-structure infections (ESTABLISH-2): A randomised, double-blind, phase 3, non-inferiority trial. Lancet Infect. Dis. 2014, 14, 696-705. [CrossRef]

10. Prokocimer, P.; De Anda, C.; Fang, E.; Mehra, P.; Das, A. Tedizolid phosphate vs linezolid for treatment of acute bacterial skin and skin structure infections: The ESTABLISH-1 randomized trial. JAMA 2013, 309, 559-569. [CrossRef] [PubMed]

11. Flanagan, S.; Bartizal, K.; Minassian, S.L.; Fang, E.; Prokocimer, P. In vitro, in vivo, and clinical studies of tedizolid to assess the potential for peripheral or central monoamine oxidase interactions. Antimicrob. Agents Chemother. 2013, 57, 3060-3066. [CrossRef] [PubMed]

12. Ong, V.; Flanagan, S.; Fang, E.; Dreskin, H.J.; Locke, J.B.; Bartizal, K.; Prokocimer, P. Absorption, distribution, metabolism, and excretion of the novel antibacterial prodrug tedizolid phosphate. Drug Metab. Dispos. 2014, 42, 1275-1284. [CrossRef] [PubMed]

13. Sudafed (pseudoephedrine hydrochloride $60 \mathrm{mg}$ ); Package Insert; Johnson \& Johnson Pacific: New South Wales, Australia, 2007.

14. Bye, C.; Hill, H.M.; Hughes, D.T.D.; Peck, A.W. A comparison of plasma levels of L(+) pseudoephedrine following different formulations, and their relation to cardiovascular and subjective effects in man. Eur. J. Clin. Pharmacol. 1975, 8, 47-53. [CrossRef] [PubMed]

15. US Food and Drug Administration Guidance for Industry. Bioavailability and Bioequivalence Studies for Orally Administered Drug Products-General Considerations. Revision 1. Available online: http:/ / www.fda.gov/ohrms/dockets/ac/03/briefing/3995B1_07_GFI-BioAvail-BioEquiv.pdf (accessed on 14 February 2017).

16. Lodise, T.P.; Fang, E.; Minassian, S.L.; Prokocimer, P.G. Platelet profile in patients with acute bacterial skin and skin structure infections receiving tedizolid or linezolid: Findings from the phase 3 ESTABLISH clinical trials. Antimicrob. Agents Chemother. 2014, 58, 7198-7204. [CrossRef] [PubMed]

17. Prokocimer, P.; Bien, P.; DeAnda, C.; Pillar, C.M.; Bartizal, K. In vitro activity and microbiological efficacy of tedizolid (TR-700) against gram-positive clinical isolates from a phase 2 study of oral tedizolid phosphate (TR-701) in patients with complicated skin and skin structure infections. Antimicrob. Agents. Chemother. 2012, 56, 4608-4613. [CrossRef] [PubMed]

18. Hardalo, C.; Lodise, T.P.; Bidell, M.; Flanagan, S.; De Anda, C.; Anuskiewicz, S.; Prokocimer, P. Clinical safety and tolerability of tedizolid phosphate in the treatment of acute bacterial skin and skin structure infections. Expert Opin. Drug Saf. 2018, 17, 359-367. [CrossRef] [PubMed]

(C) 2018 by the authors. Licensee MDPI, Basel, Switzerland. This article is an open access article distributed under the terms and conditions of the Creative Commons Attribution (CC BY) license (http://creativecommons.org/licenses/by/4.0/). 